\title{
Cryoprobe biopsy increases the diagnostic yield in endobronchial tumor lesions
}

\author{
Christian Schumann, MD, ${ }^{\mathrm{a}}$ Jürgen Hetzel, MD, ${ }^{\mathrm{c}}$ Alexander J. Babiak MD, ${ }^{\mathrm{c}}$ Tobias Merk, MD, ${ }^{\mathrm{d}}$ \\ Thomas Wibmer, MD, ${ }^{\mathrm{a}}$ Peter Möller, MD, PhD, ${ }^{\mathrm{b}}$ Philipp M. Lepper, MD, ${ }^{\mathrm{e}}$ and Martin Hetzel, $\mathrm{MD}^{\mathrm{d}}$
}

\begin{abstract}
Objective: Forceps biopsy is the standard method to obtain specimens in endoscopically visible lesions. It is common to combine forceps biopsy with cytology methods to increase the diagnostic yield. Although the flexible cryoprobe has been established for bronchoscopic interventions in malignant stenosis, the obtained biopsies, called "cryobiopsies," have not been investigated in a large cohort of patients. The aim of this feasibility study was to prospectively evaluate the diagnostic yield and safety of cryobiopsy and forceps biopsy.
\end{abstract}

Methods: During a 6-year period, 296 patients with visible endoluminal tumor lesions were included in the study at the bronchoscopy unit of a university hospital. In the first consecutively conducted 55 cases, both techniques, forceps biopsy and cryobiopsy, were applied simultaneously. Pathologic and quantitative image analyses were performed to evaluate the size and quality of the obtained specimens. We evaluated the safety and diagnostic yield to describe the feasibility of cryobiopsy.

Results: Comparative analysis of the first conducted and randomly assigned 55 cases revealed a significantly higher diagnostic yield for cryobiopsy compared with forceps biopsy $(89.1 \% \mathrm{vs} 65.5 \%, P<.05)$. In this cohort, quantitative image analysis showed significantly larger biopsies regarding size and artifact-free tissue sections for cryobiopsy compared with forceps biopsy $(P<.0001)$. The overall diagnostic yield of cryobiopsy was $89.5 \%$. Mild bleeding occurred in 11 cases $(3.7 \%)$, moderate bleeding occurred in 3 cases $(1.0 \%)$, and severe bleeding occurred in 1 case $(0.3 \%)$.

Conclusion: Cryobiopsy is safe and increases the diagnostic yield in endobronchial tumor lesions. The method also is feasible under routine conditions. (J Thorac Cardiovasc Surg 2010;140:417-21)

Diagnostic bronchoscopy with endobronchial forceps biopsy (FB) is primarily practiced in patients with suspected thoracic malignancy and visible endobronchial pathology. A comprehensive review of the literature indicates a diagnostic yield of FB of approximately $74 \%{ }^{1}$ With the forceps generally used, biopsy specimens have a diameter of approximately $2 \mathrm{~mm}^{2}$ The diagnostic utility is limited by their small size, which may result in several problems. First, there is a moderate yield of sections per biopsy specimen. Second, the mechanical damage of the biopsy specimen causes difficulties in interpretation of anatomic structure

\footnotetext{
Center of Internal Medicine, Department of Internal Medicine II, ${ }^{\mathrm{a}}$ and Department of Pathology, ${ }^{\mathrm{b}}$ University of Ulm, Ulm, Germany; Department of Internal Medicine II, ${ }^{\mathrm{c}}$ University of Tübingen, Tübingen, Germany; Clinics of Pneumology and Internal Medicine, ${ }^{\mathrm{d}}$ Red Cross Hospital, Stuttgart, Germany; and Department of Pneumology, ${ }^{\mathrm{e}}$ University Hospital of Bern (Inselspital), and University of Bern, Bern, Switzerland.

Disclosures: C.S., P.L., T.M., T.W., and P.M. have nothing to declare; M.H., A.B., and J.H. received lecture grants from ERBE, Tuebingen, Germany.

C.S. and J.H. contributed equally to the manuscript.

Received for publication Oct 12, 2009; revisions received Nov 21, 2009; accepted for publication Dec 13, 2009; available ahead of print March 12, 2010.

Address for reprints: Christian Schumann, MD, University of Ulm, Clinic of Internal

Medicine II, Albert-Einstein-Allee 23, 89081 Ulm, Germany (E-mail: christian.

schumann@uniklinik-ulm.de).

$0022-5223 / \$ 36.00$

Copyright (C) 2010 by The American Association for Thoracic Surgery

doi:10.1016/j.jtcvs.2009.12.028
}

and histopathologic changes. Finally, because vital tumor tissues do not always present further, immunohistochemical staining is hampered. The diagnostic yield can be increased by combining bronchial biopsy with complementary diagnostic methods, such as needle aspiration, bronchial lavage cytology, and brush cytology; however, this leads to a relevant increase in time and costs. ${ }^{3-8}$

The introduction of a new sampling technique is another opportunity to increase the diagnostic yield. The flexible cryoprobe is primarily used for cryoextraction of malignant airway stenosis and was introduced as an alternative method for mechanical tumor debulking, which is immediately effective. ${ }^{9,10}$ Because of extraordinarily well-preserved tissue samples (larger in size with less mechanical damage and mostly vital tumor) from cryorecanalization procedures, the technique has been transferred to the biopsy of endobronchial lesions. The aim of this study was to assess the diagnostic yield and to show the feasibility and safety of endobronchial biopsies using the flexible cryoprobe. In addition, for the new diagnostic method, the sensitivity of cryobiopsy (CB) compared with FB was evaluated.

\section{MATERIALS AND METHODS \\ Patients}

All inpatients and outpatients who were referred to Pneumology of the University Clinic of Ulm with endobronchial visible lesions and were eligible for FB or $\mathrm{CB}$ were included in the study. The first 55 patients were 


\section{Abbreviations and Acronyms \\ $\mathrm{CB}=$ cryobiopsy \\ $\mathrm{FB}=$ forceps biopsy}

randomized consecutively (prospective, poised, randomized 2-arm study) for the sequence of the biopsy procedure (FB first and $\mathrm{CB}$ second or vice versa) to exclude any interaction by the sampling method. After the interim analysis of the first 55 patients revealed the safety and efficacy of $\mathrm{CB}$, we extended the study cohort with patients in whom $\mathrm{CB}$ was performed under routine conditions but who were not randomized for the sequence of the biopsy procedure. During a period of 6 years, 296 patients were included in the study. The inclusion and exclusion criteria were as follows:

\section{Inclusion Criteria}

- Exophytic endobronchial tumor (endoscopically visible lesion)

- Signed informed consent form in the first cohort

- Sufficient respiratory function (oxygen saturation $>90 \%$ with 2 liters of supplementary oxygen)

\section{Exclusion Criteria}

- Previous cancer-specific treatment or endobronchial diagnostic procedure

- Suspected connection of the lesion to large pulmonary blood vessels as seen on chest computed tomography scan

- Thrombocyte count less than $100 \mathrm{G} / \mathrm{L}$ and abnormal plasma clotting

- Age less than 18 years

\section{Bronchoscopy}

All interventions primarily were done with the flexible technique; however, the rigid scope (in general anesthesia) was used if centrally located lesions indicated further procedures, such as recanalization or stent implantation. After sedation with midazolam or propofol (Disoprivan, AstraZeneca, Wilmington, DE), patients were intubated using a flexible endotracheal tube (Bronchoflex, 7.5 mm, Rüsch GmbH, Kernen, Germany), but spontaneous breathing was maintained throughout the procedure. Before biopsy sampling, a bronchoscopic assessment of patients' bronchial systems was performed. Continuous monitoring of oxygen saturation, heart rate, and blood pressure was performed.

\section{Cryoprobe}

The flexible cryoprobe is $780 \mathrm{~mm}$ in length and $2.3 \mathrm{~mm}$ in diameter. The instrument (Erbokryo, ERBE Medizintechnik GmbH, Tuebingen, Germany) uses nitrous oxide and achieves a temperature of $-89.5^{\circ} \mathrm{C}$ at the tip of the probe's gas channel. The cryoprobe is a closed system that can be resterilized and reused.

\section{Forceps}

FB was performed using a reusable fenestrated forceps FB-21C or FB52C-1 (Olympus Corp, Hamburg, Germany).

\section{Biopsy Procedures}

At least 2 biopsy specimens were obtained by the cryoprobe. Both techniques, FB and $\mathrm{CB}$, were applied simultaneously only in the first 55 patients (randomized cohort). These patients were randomly assigned to the order of the biopsy technique to exclude any interaction by the sampling method. In these cases, 2 biopsy specimens were taken from the exophytic tumor by each method. The following FB was performed conventionally, whereas $\mathrm{CB}$ was performed as follows: Guided through the bronchoscope's working channel, the flexible cryoprobe was placed onto the endobronchial lesion. The freezing process was started for 3 seconds or until the ice front reached the bronchial wall. After 3 seconds, the probe was pulled out together with the bronchoscope and the frozen tissue sample on the probe's tip. The tissue was then released from the probe's tip by thawing in a sterile $0.9 \%$ sodium chloride water bath.

\section{Pathology}

All obtained specimens were immediately fixed in buffer formalin for up to 24 hours. The paraffin-embedded sections were taken and processed for staining with hematoxylin-eosin. An institutional pathologist routinely conducted the histologic analysis.

\section{Quantitative Image Analysis}

Specimens from patients who received both biopsy procedures underwent quantitative image analysis to compare the size and quality. The sections with the least damage and best preservation were selected for quantitative image analysis. Slides were analyzed with a Zeiss Interactive Analysis System (Carl Zeiss AG, Oberkochen, Germany). Components of the system used and its processing are as follows: A Zeiss Axiophot light microscope was connected to a Kontron Interactive Analysis System (Eching, Germany) image analyzer with a charged-coupled device camera. The outer boundaries of the biopsy particles were traced interactively with a mouse on a graphic tablet under visual control of the microscopic image on the screen at low-power magnification. Contaminations by blood were excluded from the measurement. The total area of the biopsy particles was measured in millimeters squared.

\section{Diagnosis}

For each patient, a definitive diagnosis was made on the basis of the results of the pathology and other findings, for example, patient history, clinical examination, laboratory findings, and radiology. The diagnosis corresponded with the routinely generated doctor's report.

\section{Safety}

Safety data were evaluated regarding severity of bleeding, postinterventional pneumothorax, or pneumomediastinum. In advance of any intervention, patients were checked to have a platelet count of at least $100 \mathrm{G} / \mathrm{L}$ and normal coagulation parameters (partial thromboplastin time $<40$ seconds and prothrombin ratio $>70 \%$ ). Medications influencing the clotting time were stopped before intervention: heparin for at least 24 hours and inhibitors of platelet aggregation for at least 7 days. Bleeding was graded as mild if cold water $\left(2^{\circ} \mathrm{C}-4^{\circ} \mathrm{C}\right)$ or epinephrine solution $(1 \mathrm{mg} / 10 \mathrm{~mL}$ saline water $)$ was necessary to stop bleeding, moderate if additional techniques (argon plasma coagulator, bronchus blocker) were used, and severe if additional systemic treatment was needed (transfusion of red blood cells, platelets, fresh-frozen plasma or coagulation factors, fluid resuscitation, use of vasopressors) to stabilize a patient's condition. No or minimal bleeding was bleeding that did not require any of the above-mentioned measures (ie, suctioning only).

\section{Statistical Analysis}

For quantitative image analysis data, mean, standard deviation, and standard error of mean were determined. To compare differences of the sample size between FB and CB, the Wilcoxon signed-rank test was used. To determine the diagnostic yield of each sampling method, their pathologic findings were compared with final diagnosis. Contingency tables and Fisher's exact test were used to compute significances. 


\section{RESULTS \\ Patient Data}

Over a period of 6 years, we included 296 patients (male/ female: $225 / 71$; age, mean \pm standard deviation, range: $63.4 \pm 11.8,20-84$ years; inpatients/outpatients: 179/117) with bronchoscopic diagnostic intervention who received a $\mathrm{CB}$ in the final analysis. All patients had visible lesions. From 236 cases $(79.7 \%)$ with malignancy, 196 cases $(83.1 \%)$ had lung cancer (Table 1$)$.

\section{Interventions}

All interventions were performed with the flexible technique. The rigid scope was also used in 17 patients during subsequent therapeutic intervention (recanalization, stent implantation).

\section{Efficacy}

Evaluation of the first 55 patients (randomized cohort) revealed an advantage for the new technique over conventional FB. CB led to the final diagnosis in 49 of 55 patients $(89.1 \%)$, and FB led to the final diagnosis in 36 of 55 patients $(65.5 \%)$. The achieved diagnostic yield for $\mathrm{CB}$ was significantly higher than for FB $(P<.05)$, as shown in Figure 1, $A$. Subgroup analysis showed no influence on these results by the sampling order or by localization of the tumor lesion within the bronchial tree.

TABLE 1. Overview of histologic findings

\begin{tabular}{lc}
\hline \multicolumn{1}{c}{ Conditions } & Patients, no. (\%) \\
\hline Nonmalignant lesions & $60(20.3)$ \\
Malignant lesions & $236(79.7)$ \\
Lung cancer & $196(83.1)$ \\
$\quad$ SCLC & $46(23.5)$ \\
$\quad$ NSCLC & $150(76.9)$ \\
$\quad$ Adenocarcinoma (including BAC) & $29(19.3)$ \\
$\quad$ Squamous cell cancer & $86(57.3)$ \\
$\quad$ Large cell lung cancer & $4(2.7)$ \\
$\quad$ not specified NSCLC/mixed type & $29(19.3)$ \\
$\quad$ Carcinoma in situ & $2(1.3)$ \\
Colorectal cancer & $7(3.0)$ \\
Carcinoids & $6(2.6)$ \\
Lymphoma & $4(1.7)$ \\
Breast cancer & $8(3.4)$ \\
Lung carcinosarcoma & $3(1.3)$ \\
Hepatocellular carcinoma & $1(0.4)$ \\
Renal cell carcinoma & $3(1.3)$ \\
Pancreatic cell carcinoma & $1(0.4)$ \\
Prostate cancer & $1(0.4)$ \\
Laryngo-pharyngeal carcinoma & $4(1.7)$ \\
Urinary bladder carcinoma & $1(0.4)$ \\
Malignant pleural mesothelioma & $1(0.4)$ \\
\hline NCC, Non-small cell & $B C .670 n c-$
\end{tabular}

$N S C L C$, Non-small cell lung cancer; $S C L C$, small cell lung cancer; $B A C$, bronchioloalveolar carcinoma.
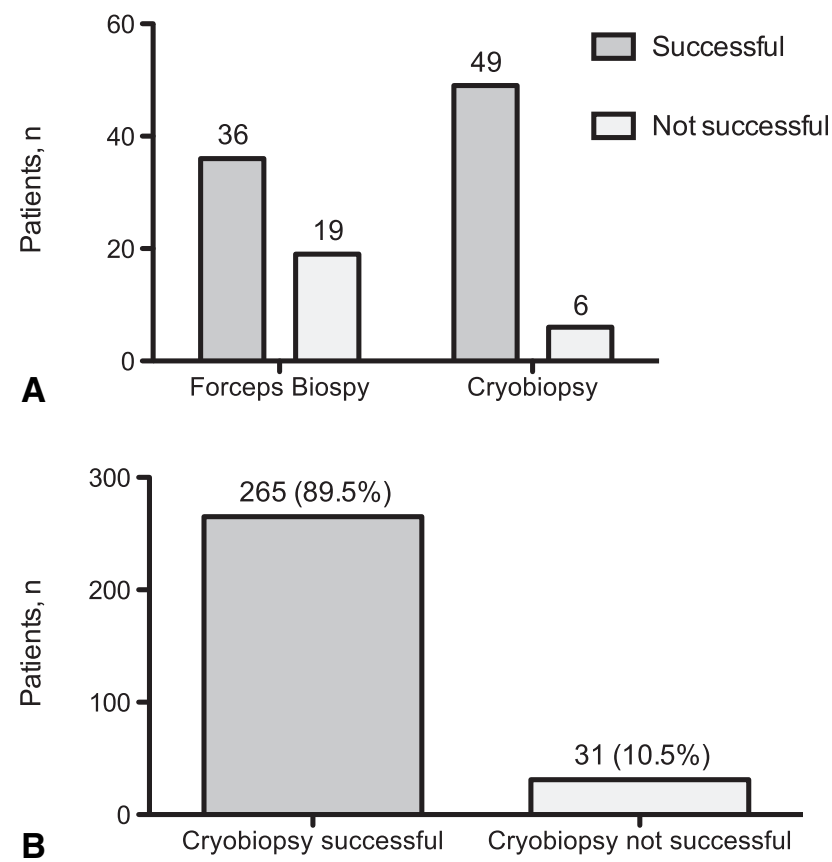

FIGURE 1. A, Diagnostic yield of CB versus FB in randomized cohort of 55 patients. Graph shows results from contingency table analysis with $P<.05$ (Fisher's exact test). B, Efficacy of CB in the overall cohort of 295 patients.

Evaluation of all patients revealed that $\mathrm{CB}$ was successful in 265 of 296 cases and led to a diagnosis in $89.5 \%$ of patients (Figure 1, B). Further evaluations regarding subgroup differences (non-small cell lung cancer vs small cell lung cancer; adenocarcinoma vs squamous cell carcinoma) showed an additional advantage for CB.

\section{Quantitative Image Analysis}

The mean total area of each tissue section in the CB slides was $10.4 \mathrm{~mm}^{2}$ and thus was significantly larger than that of $\mathrm{FB}$, with a mean total area of $5.2 \mathrm{~mm}^{2}(P<.0001)$. Furthermore, the artifact-free tissue areas of each slide were also significantly greater with $\mathrm{CB}$ than with FB (9.6 vs $3.6 \mathrm{~mm}^{2}, P<.0001$, Figure 2).

\section{Safety}

Notable bleeding complications occurred in 15 cases $(5.1 \%)$. These were regarded as mild bleeding in 11 cases $(3.7 \%)$, moderate bleeding in 3 cases $(1.0 \%)$, and severe bleeding in 1 case $(0.3 \%)$, which occurred in a 78 -yearold patient with a single visible lesion in the right upper lobe. Because of prolonged bleeding during intervention with the cryoprobe, supposing an affected tumor vessel, transfusion of 1 red blood cell concentrate was performed. Subsequently, the patients fully recovered from the intervention. Thus, the overall complication rate was low. 


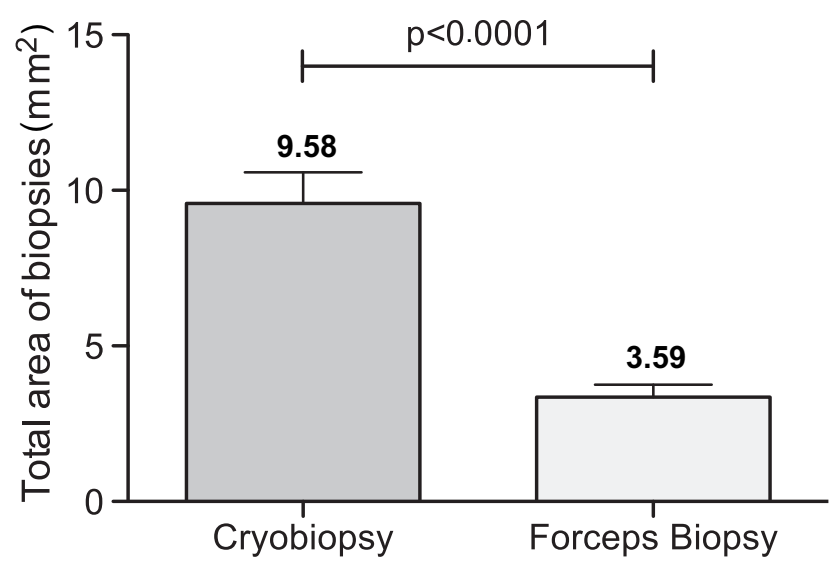

FIGURE 2. Artifact-free tissue areas of $\mathrm{CB}$ versus $\mathrm{FB}$ in randomized cohort of 55 patients.

\section{DISCUSSION}

In patients with endoscopically visible lesions, flexible bronchoscopy with FB is the most frequently used technique to obtain specimens for pathologic analysis. The sensitivity of FB is approximately $74 \%$. Only a combination of cytologic methods (brushing, washing, and needle) might increase the yield up to $89.5 \%$, but this is associated with a relevant increase in procedural time and costs. On the other hand, costs to establish a new method have to be taken into account. The flexible cryoprobe, so far used for cryotherapy and endoluminal tumor debulking, also seemed to be suitable for biopsies from visible lesions. The purpose of our study was to assess the efficacy of CB and to compare it with the standard method of FB. Furthermore, we aimed to show the feasibility of this new technique in a larger population under routine conditions.

\section{Patient Data}

The mean age, gender, and distribution of histologic subtypes with a majority of squamous cell lung cancer $(57.3 \%)$ were as expected.

\section{Efficacy}

Our data confirm the findings of previous studies that the sensitivity of FB is approximately $74 \% .^{1}$ The diagnostic yield of FB in our study was $65.5 \%$, somewhat lower as assumed. Compared with other studies that used multiple sampling ( $\sim 3-4$ specimens), only 2 specimens were obtained from every lesion, according to our study protocol. Multiple sampling may slightly increase the yield and explain these minimal differences. Gellert and colleagues ${ }^{11}$ showed that at least 5 biopsy specimens were required to provide a greater than $90 \%$ probability of obtaining at least 1 positive sample. Because the results of the interim analysis of the first included 55 patients (who were randomly assigned to the order of the sampling method) showed that the diagnostic yield of CB was significantly higher compared with FB (89.1\%,
$P<.05$ ), we used the cryoprobe routinely for biopsies from endobronchial visible lesions and extended the patient cohort. The overall diagnostic yield of $89.5 \%$ from all 296 patients during a 6-year period proved our initial findings. These data show that $\mathrm{CB}$ is feasible with a consistently high diagnostic yield under routine conditions.

The quantitative image analysis showed an advantage for CB over FB. CB had significantly larger mean total tissue area $\left(10.4\right.$ vs $\left.5.2 \mathrm{~mm}^{2} ; P<.0001\right)$ and artifact-free tissue area $\left(9.6 \mathrm{vs} 3.4 \mathrm{~mm}^{2} ; P<.0001\right)$ per slide, indicating the higher quality of $\mathrm{CB}$. Generally, it is without doubt that the bigger the FB, the larger the obtained specimens, and better tissue quality leads to better results. But studies that used different types of forceps to compare the obtained specimens have provided controversial results. ${ }^{2}$ Even if specimens were larger, the mechanical damage significantly influenced the results. Our results demonstrate that CB leads to not only larger but also qualitatively better specimens. This is mainly achieved by markedly less mechanical damage of the specimens obtained with the cryoprobe. There are 3 main reasons for the improved quality of CB. First, during the sampling procedure the cryoprobe only needs to gently touch the tumor lesion (without any squeezing, unlike in the use of FB), which leads to less mechanical damage. Second, the application of up to $-89^{\circ} \mathrm{C}$ at the probe's tip has hemostatic effects in the tissue, which results in artifactfree tissue. Finally, the specimens are extracted in a freshfrozen condition. These 3 factors result in larger tumor tissue per section and in better possibilities for evaluation by the pathologist. Neither the sampling order nor the localization of the suspected lesion within the bronchial tree significantly influenced the higher diagnostic yield of CB.

\section{Safety}

The bleeding rate of FB is assumed to be generally low. In previous studies, the bleeding rates vary from 1 major complication after FB (an endobronchial visible carcinoma) to 11 potentially life-threatening hemorrhages after FB or to a bleeding rate of up to $17 \%$ with FB. A recent study using "hot" biopsy by electrocoagulation showed a reduced bleeding rate without a negative impact on the pathologic samples. ${ }^{12}$ In contrast with these results, a "cold" CB increased the diagnostic yield significantly with comparably low bleeding rates.

\section{CONCLUSIONS}

$\mathrm{CB}$ is a safe and feasible method with a bleeding rate less than or comparable to that of $\mathrm{FB}$. CB has a significantly higher diagnostic yield than FB, mainly because of the larger size and better tissue quality of the obtained specimens. Especially in the era of targeted therapy, larger and better tumor tissues are of high value for further immunohistochemical staining procedures and comprehensive molecular 
analysis. An important advantage of $\mathrm{CB}$ is that advanced pathologic methods are not limited to small sample size.

\section{References}

1. Schreiber G, McCrory DC. Performance characteristics of different modalities for diagnosis of suspected lung cancer: summary of published evidence. Chest. 2003; 123(1 Suppl):115S-28S.

2. Aleva RM, Kraan J, Smith M, ten Hacken NH, Postma DS, Timens W. Techniques in human airway inflammation: quantity and morphology of bronchial biopsy specimens taken by forceps of three sizes. Chest. 1998;113:182-5.

3. Bilaceroglu S, Gunel O, Cagirici U, Perim K. Comparison of endobronchial needle aspiration with forceps and brush biopsies in the diagnosis of endobronchial lung cancer. Monaldi Arch Chest Dis. 1997;52:13-7.

4. Dasgupta A, Jain P, Minai OA, Sandur S, Meli Y, Arroliga AC, et al. Utility of transbronchial needle aspiration in the diagnosis of endobronchial lesions. Chest. 1999;115:1237-41.

5. Govert JA, Dodd LG, Kussin PS, Samuelson WM. A prospective comparison of fiberoptic transbronchial needle aspiration and bronchial biopsy for bronchoscopically visible lung carcinoma. Cancer. 1999;87:129-34.
6. Govert JA, Kopita JM, Matchar D, Kussin PS, Samuelson WM. Cost-effectiveness of collecting routine cytologic specimens during fiberoptic bronchoscopy for endoscopically visible lung tumor. Chest. 1996;109:451-6.

7. Lam WK, So SY, Hsu C, Yu DY. Fibreoptic bronchoscopy in the diagnosis of bronchial cancer: comparison of washings, brushings and biopsies in central and peripheral tumours. Clin Oncol. 1983;9:35-42.

8. Schenk DA, Bryan CL, Bower JH, Myers DL. Transbronchial needle aspiration in the diagnosis of bronchogenic carcinoma. Chest. 1987;92:83-5.

9. Hetzel M, Hetzel J, Schumann C, Marx N, Babiak A. Cryorecanalization: a new approach for the immediate management of acute airway obstruction. $J$ Thorac Cardiovasc Surg. 2004;127:1427-31.

10. Schumann C, Lepper PM, Barth TF, Moller P, Kruger S. Successful immediate cryorecanalization of a simultaneous high-grade tracheal and bronchial stenosis as rare manifestations of bronchial-associated lymphoid tissue lymphoma J Thorac Cardiovasc Surg. 2009;137:e17-9.

11. Gellert AR, Rudd RM, Sinha G, Geddes DM. Fibreoptic bronchoscopy: effect of multiple bronchial biopsies on diagnostic yield in bronchial carcinoma. Thorax. 1982;37:684-7.

12. Tremblay A, Michaud G, Urbanski SJ. Hot biopsy forceps in the diagnosis of endobronchial lesions. Eur Respir J. 2007;29:108-11. 